This is an electronic reprint of the original article. This reprint may differ from the original in pagination and typographic detail.

Author(s): Herranen, Matti; Hohenegger, Andreas; Osland, Asgeir; Tranberg, Anders

Title: Quantum corrections to inflation : The importance of RG running and choosing the optimal RG scale

Year: $\quad 2017$

Version:

Please cite the original version:

Herranen, M., Hohenegger, A., Osland, A., \& Tranberg, A. (2017). Quantum corrections to inflation : The importance of RG running and choosing the optimal RG scale. Physical Review D, 95(2), Article 023525.

https://doi.org/10.1103/PhysRevD.95.023525

All material supplied via JYX is protected by copyright and other intellectual property rights, and duplication or sale of all or part of any of the repository collections is not permitted, except that material may be duplicated by you for your research use or educational purposes in electronic or print form. You must obtain permission for any other use. Electronic or print copies may not be offered, whether for sale or otherwise to anyone who is not an authorised user. 


\title{
Quantum corrections to inflation: The importance of RG running and choosing the optimal RG scale
}

\author{
Matti Herranen* \\ Department of Physics, P.O.Box 35 (YFL), FI-40014 University of Jyväskylä, Jyväskylän yliopisto, Finland
}

Andreas Hohenegger, ${ }^{\dagger}$ Asgeir Osland, ${ }^{\dagger}$ and Anders Tranberg ${ }^{\S}$

Faculty of Science and Technology, University of Stavanger, N-4036 Stavanger, Norway

(Received 10 October 2016; published 31 January 2017)

\begin{abstract}
We demonstrate the importance of correctly implementing RG running and choosing the RG scale when calculating quantum corrections to inflaton dynamics. We show that such corrections are negligible for single-field inflation, in the sense of not altering the viable region in the $n_{s}-r$ plane, when imposing Planck constraints on $A_{s}$. Surprisingly, this also applies, in a nontrivial way, for an inflaton coupled to additional spectator degrees of freedom. The result relies on choosing the renormalization scale (pseudo-) optimally, thereby avoiding unphysical large logarithmic corrections to the Friedmann equations and large running of the couplings. We find that the viable range of parameters of the potential is altered relative to the classical limit, and we find an upper limit of $g \simeq 10^{-4}$ on the value of the inflaton-spectator portal coupling still allowing for inflation and an upper limit of $g \simeq 10^{-5}$ for inflation to correctly reproduce the scalar amplitude of fluctuations $A_{s}$.
\end{abstract}

DOI: $10.1103 /$ PhysRevD.95.023525

\section{INTRODUCTION}

Observations of the cosmic microwave background indicate a period of accelerated expansion in the early Universe, known as inflation. The predominant theoretical realization of such an epoch is through the potential energy of a slowly rolling scalar field, the inflaton. The choice of the potential function $V(\phi)$ largely determines the observable spectrum of scalar and tensor metric fluctuations.

Traditionally, model building and predictions rely on the classical dynamics of a homogeneous field in an expanding background metric, and in many cases the simplification of expressing evolution equations in terms of a set of slow-roll parameters, equations that may then be truncated at some order in powers of these parameters. But ultimately, this homogeneous field must be identified as the quantum expectation value of a quantum field $\hat{\phi}$ (1-point function, mean field, condensate), subject to a complete quantum field theoretical treatment. Then, $V(\phi)$ must be identified as the quantum effective potential. ${ }^{1}$

Various choices of $V(\phi)$ in model building must therefore ideally be connected to an underlying quantum theory, restricting the form that such a potential may take. As an example, it is well known that an interacting theory at tree

\footnotetext{
*matti.h.herranen@jyu.fi

†andreas.hohenegger@uis.no

*asgeir.osland@uis.no

§anders.tranberg@uis.no

${ }^{1}$ This is not the low-energy effective potential, obtained from integrating out heavier degrees of freedom, but the quantum effective potential from integrating out all fluctuations and is a function of the mean field only.
}

level generates a tower of interactions at loop level and that the couplings run with the renormalization scale. In particular, nonminimal coupling to gravity is generated away from the conformal limit, and field self-interactions generically generate logarithmic dependence on the field.

Quantum corrections are often argued to be "small" in some sense and can be controlled by powers of the couplings. On the other hand, there are models in which the required small inflaton mass arises as a quantum effect. The responsible couplings can also cause a strong scale-dependent running of this mass parameter [1-3]. Otherwise, for singlefield inflation, the self-interactions are typically small to provide the necessary flatness of the potential during the inflationary epoch. However, the renormalization scale is a priori arbitrary, and it is tempting to choose it to augment the effects of running [4,5]. This misses the point that the renormalization scale is a parametrization of a perturbative diagram truncation. A large dependence on this scale is a signal that perturbation theory is unreliable, and so to trust the computation, it is no longer arbitrary, but subject to a criterion of "small dependence".

Quantum corrections to inflation has been considered several times before (see, for instance, [4-23] for a broad range of approaches), with some works reporting significant quantum effects and some negligible effects. Because taking all aspects of the calculation into account is quite involved, often focus is put on a few such aspects, while neglecting or ignoring others (typical examples are the inclusion or omission of RG running, curvature effects, IR effects and resummations, scalar metric fluctuations). Often, a slow-roll treatment is introduced, which requires that the effective potential in the inflation equation of 
motion is the same as in the Friedmann equation. This is, in general, not the case (see, for instance, [21]).

In models with additional symmetry (SUSY being the obvious example, see, for instance, [24,25]), quantum corrections may be very small as a result of (approximate) cancellations. Similarly, running of the couplings may be absent altogether. Invoking such symmetries is an elegant model-building tool not to spoil the flatness of the potential (and hence inflation) at the quantum level. It is complementary to the scope of the present paper.

In this work, we consider two models of inflation: one with a single self-interacting field and one where this single field is coupled to a spectator field. We compute the 1-loop effective potential and 1-loop (or 2-loop) RG running, including curvature effects, but neglect IR effects. The size of the quantum contributions, throughout the evolution, depends manifestly on the RG scale. As we explain, there is no truly optimal choice of this scale for the second model, but our results suggest one that is preferred, by far, over keeping it constant. By performing comprehensive parameter scans using a modified version of multimodecode $[26],{ }^{2}$ we investigate for both models whether, when using a (pseudo-)optimal, time-dependent RG scale, quantum corrections may still be important. We find that for a single inflaton field, the smallness of the self-interaction makes the corrections negligible. But, when coupled to other fields, the size of that coupling is a priori unconstrained (within reason), potentially leading to a significant modification in the basic CMB predictions. However, we see that while this is true for the individual trajectory, the overall region of $n_{s}-r$ produced by these inflation models are largely unchanged when including quantum corrections.

As for many other works on this subject, we also rely on the "semi-classical" approximation to quantum corrections in curved space-time, where the scalar metric fluctuations are ignored. The quantum scalar field(s) evolve in a classical Friedmann-Robertson-Walker (FRW) background. Including metric fluctuations is discussed in some details in [23], where we argue that for large-field inflation as considered in the present work, the semiclassical approach may not capture all the relevant quantum corrections. Hence, finding large semiclassical corrections would motivate a somewhat more involved computation including metric fluctuations.

The structure of the paper is as follows: In Sec. II, we introduce the single-field model, slow-roll, and quantum corrections as well as the RG running and show how to choose the RG scale. In Sec. III, this model is subject to a numerical sampling and computation of the CMB spectrum including quantum corrections. We analyze the magnitude of the corrections from their projection onto the $n_{s}-r$ plane. In Sec. IV, we extend the single-field model with a

\footnotetext{
${ }^{2}$ This does not rely on a slow-roll approximation for solving the field dynamics.
}

"portal" coupling to a second scalar in its vacuum and describe again the quantum corrections, RG running, and one convenient choice of RG scale. Section V is then a numerical sampling of the resulting model. We again consider the impact on the observationally allowed region in an $n_{s}-r$-diagram and analyze to what extent our convenient choice of RG scale is optimal. Section VI is a technical description of the numerical procedure and modifications to Multimodecode, an existing off-the-shelf package underlying our code. We conclude in Sec. VII.

\section{ONE SELF-INTERACTING, MASSIVE FIELD, NONMINIMALLY COUPLED TO GRAVITY}

We consider a single-field inflation model, with the action

$$
\begin{aligned}
S= & \int d^{n} x \sqrt{-g} \\
& \times\left[\frac{M_{\mathrm{pl}}^{2}}{2} f(\phi) R+\frac{1}{2} \partial_{\mu} \phi \partial^{\mu} \phi-\frac{1}{2} m^{2} \phi^{2}-\frac{\lambda}{24} \phi^{4}\right]
\end{aligned}
$$

and where we choose the specific case

$$
f(\phi)=1-\frac{\xi \phi^{2}}{M_{\mathrm{pl}}^{2}} .
$$

This is the simplest and most common nonminimal coupling between gravity and matter.

\section{A. Classical slow-roll}

For a nonminimally coupled theory, the usual slow-roll parameters

$$
\epsilon_{1}=-\frac{\dot{H}}{H^{2}}, \quad \epsilon_{2}=-\eta=\frac{\ddot{\phi}}{H \dot{\phi}},
$$

must be supplemented by (see, for instance, [27-32])

$$
\epsilon_{3}=-\frac{\xi \phi \dot{\phi}}{H M_{\mathrm{pl}}^{2} f(\phi)}, \quad \epsilon_{4}=\frac{6 \xi\left(\xi-\frac{1}{6}\right) \phi \dot{\phi}}{H\left(M_{\mathrm{pl}}^{2}+6 \xi\left(\xi-\frac{1}{6}\right) \phi^{2}\right)} .
$$

When all of these are $\ll 1$, the Universe is inflating, and the inflaton field is slow-rolling. It then makes sense to write down and expand the equations of motion and observables in powers of $\epsilon_{1,2,3,4}$. In a flat FRW background, the classical field equation of motion then reads

$$
3 H \dot{\phi}\left(1+\frac{1}{3} \epsilon_{2}\right)=-\left(m^{2}+\xi R\right) \phi+\frac{1}{6} \lambda \phi^{3},
$$

with the scalar curvature $R=12 H^{2}+6 \dot{H}=12 H^{2}\left(1-\epsilon_{1} / 2\right)$. This follows from the variation of the action with respect to the field $\phi$. The Friedmann equations follow from variation of the action with respect to a general metric $g^{\mu \nu}$ and subsequently specializing to FRW space. We find 


$$
\begin{gathered}
3 M_{\mathrm{pl}}^{2} H^{2} f(\phi)\left(1+2 \epsilon_{3}\right) \\
=\frac{1}{2} \dot{\phi}^{2}+\frac{1}{2} m^{2} \phi^{2}+\frac{\lambda}{24} \phi^{4}, \\
3 M_{\mathrm{pl}}^{2} H^{2} f(\phi)\left(\frac{2}{3} \epsilon_{1}-1-\frac{4}{3} \epsilon_{3}\left(1+\frac{1}{2} \epsilon_{2}\right)\right) \\
=\frac{1}{2}(1-4 \xi) \dot{\phi}^{2}-\frac{1}{2} m^{2} \phi^{2}-\frac{\lambda}{24} \phi^{4} .
\end{gathered}
$$

In addition, we define the quantity

$$
\delta=\frac{V^{\prime \prime}}{H^{2}}=\frac{m^{2}+\xi R+\frac{1}{2} \lambda \phi^{2}}{H^{2}}
$$

which, for a one-field model of inflation, is related to leading order in slow-roll as $\delta \simeq 3\left(\epsilon_{2}-\epsilon_{1}\right)$. In the slow-roll limit (i.e., neglecting $\epsilon_{1,2,3,4}$ relative to constant of order 1 ), the field and Friedmann equations become

$$
\begin{gathered}
3 H \dot{\phi}=-\left[\left(m^{2}+12 \xi H^{2}\right) \phi+\frac{1}{6} \lambda \phi^{3}\right], \\
3 M_{\mathrm{pl}}^{2} H^{2} f(\phi)=\frac{1}{2} m^{2} \phi^{2}+\frac{\lambda}{24} \phi^{4} .
\end{gathered}
$$

The standard procedure is then to define the end of inflation as $\epsilon_{1}=1$, back-track the evolution of $\phi$ and $H$ a time corresponding to $N e$-folds, and compute the basic CMB observables at this "horizon crossing epoch". These are for a nonminimally coupled model given by

$$
\begin{gathered}
A_{s}=\frac{1}{4 \pi^{2}} \frac{H^{4}}{\dot{\phi}^{2}}, \\
n_{s}-1=-4 \epsilon_{1}-2 \epsilon_{2}+2 \epsilon_{3}-2 \epsilon_{4}, \\
r=16\left(\epsilon_{1}+\epsilon_{3}\right),
\end{gathered}
$$

for the pivot-scale amplitude $A_{s}$, scalar spectral index $n_{s}$ and scalar-to-tensor ratio of amplitudes $r$, respectively. Results based on solving (5), (6), and (7) we refer to as classical, to distinguish them from the quantum corrected evolution we introduce in the following. Similarly, (9) and (10) denote classical slow-roll. The expressions of the central observables, Eqs. (11), (12), and (13) follow from the evolution of the quantum modes of the metric fluctuations. For the 1-loop, 1PI quantum treatment we perform in the following, they are unaltered as expressions in slow-roll parameters. They may of course take on different values in case the quantum corrected dynamics produces trajectories in $(\phi, \dot{\phi}, H, \dot{H})$ different from the classical ones.

\section{B. Quantum corrections}

In the semiclassical approach, we may straightforwardly compute the 1-loop effective potential in an FRW background (see, for instance, [18]) we find in the $\overline{\mathrm{MS}}$ scheme

$$
\begin{aligned}
V_{\text {eff }}= & \frac{1}{2} m^{2}(\mu) \phi^{2}(\mu)+\frac{1}{2} \xi(\mu) R \phi^{2}(\mu) \\
& +\frac{1}{24} \lambda(\mu) \phi^{4}(\mu)+\frac{1}{64 \pi^{2}} M^{4}(\phi)\left[\log \frac{\left|M^{2}(\phi)\right|}{\mu^{2}}-\frac{3}{2}\right] .
\end{aligned}
$$

We have introduced the mass (squared) $M^{2}(\phi)$, which appears in the conformal-time field mode equation. ${ }^{3}$ It reads

$$
M^{2}(\phi)=m^{2}(\mu)+\frac{1}{2} \lambda(\mu) \phi^{2}(\mu)+\left(\xi(\mu)-\frac{1}{6}\right) R .
$$

The couplings $m^{2}(\mu), \lambda(\mu), \xi(\mu)$, and the field $\phi(\mu)$ are now running with the renormalization scale $\mu$, relative to some reference scale $\mu_{0}$. In the following, we suppress the explicit $\mu$ in our notation, but keeping in mind that whenever quantum corrections are included, all the parameters run with this scale. In the classical approximation, they do not. By variation of the effective potential, we find the equation of motion for the mean field

$$
\begin{aligned}
\ddot{\phi} & +3 H \dot{\phi}+\left(m^{2}+\xi R\right) \phi+\frac{1}{6} \lambda \phi^{3} \\
& +\frac{\lambda \phi M^{2}(\phi)}{32 \pi^{2}}\left(\log \frac{\left|M^{2}(\phi)\right|}{\mu^{2}}-1\right)=0 .
\end{aligned}
$$

This is an equation for the evolution of the renormalized field $\phi$ and is expressed only in terms of RG-improved couplings.

The Friedmann equations do not follow from variation of the effective action (14) but from variation of the classical action and, again in the semiclassical approach, computing the appropriate quantum expectation values [23]. The result is

$$
\begin{aligned}
3 M_{\mathrm{pl}}^{2} H^{2} & =T_{00}^{C}+T_{00}^{Q}, \\
a^{2} 3 M_{\mathrm{pl}}^{2} H^{2}\left(\frac{2}{3} \epsilon_{1}-1\right) & =T_{i i}^{C}+T_{i i}^{Q},
\end{aligned}
$$

where $T_{\mu \nu}^{C}$ denotes the classical energy-momentum tensor, and the quantum correction is

\footnotetext{
${ }^{3}$ It is not the second derivative of the potential.
} 


$$
\begin{aligned}
T_{\mu \nu}^{Q}= & -g_{\mu \nu} \frac{H^{4}}{64 \pi^{2}}\left(\log \frac{\left|M^{2}(\phi)\right|}{\mu^{2}}-1\right) \\
& \times\left[\delta^{2}-4 \delta \epsilon_{1}-2 \delta-6 \epsilon_{1}+12 \xi\left(2-\delta+\epsilon_{1}-\delta \epsilon_{1}\right)\right] .
\end{aligned}
$$

Since the object $\delta$ is not a priori a slow-roll parameter, for illustration we have included contributions to leading order in $\epsilon_{1}$ and all orders in $\delta$. From now on, $\phi$ is taken to be responsible for the $\mathrm{CMB}$ fluctuations, in which case it is a light field in the sense that $\delta$ can be considered leading order in slow-roll. Hence, in the following, we discard three of the eight terms in (18) as higher order in slow-roll $\left(\delta^{2},-4 \delta \epsilon_{1}, 12 \xi \delta \epsilon_{1}\right)$.

\section{RG running and the choice of RG scale}

The RG-improved couplings $\lambda, \mathrm{m}^{2}$, and $\xi$ follow from solving the 2-loop ${ }^{4}$ RG equations $[4,5]$

$$
\begin{gathered}
\frac{1}{\mu} \frac{d \lambda}{d \mu}=\frac{3 \lambda^{2}}{(4 \pi)^{2}}\left(1-\frac{17}{9} \frac{\lambda}{(4 \pi)^{2}}\right), \\
\frac{1}{\mu} \frac{d m^{2}}{d \mu}=m^{2} \frac{\lambda}{(4 \pi)^{2}}\left(1-\frac{5}{6} \frac{\lambda}{(4 \pi)^{2}}\right), \\
\frac{1}{\mu} \frac{d \xi}{d \mu}=\left(\xi-\frac{1}{6}\right) \frac{\lambda}{(4 \pi)^{2}}\left(1-\frac{5}{6} \frac{\lambda}{(4 \pi)^{2}}\right)+\frac{\lambda^{2}}{18(4 \pi)^{4}} .
\end{gathered}
$$

The solution is not easily written in closed form, and although we include it in our numerical integration, some of the analysis below is performed at 1-loop for illustration. The renormalized field $\phi$ is also a function of the scale through the anomalous dimension

$$
\gamma=\frac{\lambda^{2}}{12(4 \pi)^{4}}
$$

by means of wave-function renormalization:

$$
\phi(\mu)=\phi_{c} e^{-\int_{\mu_{0}}^{\mu} \frac{d \mu^{\prime} \gamma\left(\mu^{\prime}\right)}{\mu^{\prime}}}
$$

Since the action and dynamics are expressed entirely in terms of the renormalized field, the "classical" field $\phi_{c}$ will not enter explicitly.

It remains to choose the renormalization scale $\mu$ and the reference scale $\mu_{0}$. One option is to choose a fixed renormalization scale, constant in time. This is formally completely valid but may not provide a good approximation of the effective potential for all times. Since the exact effective potential is independent of the choice of $\mu$, any large dependence on this parameter is a sign that the perturbative

\footnotetext{
${ }^{4}$ Note that in the third equation for $\xi$, the 2-loop contribution is the sum of terms proportional to $\lambda^{2}$.
}

truncation is unreliable. The prescription is therefore that one should at each time choose $\mu$ so that the result depends as little as possible on its exact value. In particular, $\mu$ can be time-, field-, and/or Hubble-rate dependent.

We do the next best thing and define

$$
\mu^{2}=\frac{\left|M^{2}(\phi)\right|}{e}=\frac{\left|M^{2}\left(\phi, H, \lambda, m^{2}, \xi\right)\right|}{e} .
$$

This is highly convenient, since the Coleman-Weinberg contributions to both mean field and the entire quantum correction to the Friedman equations simply vanish. The only remaining effect of quantum corrections is that the couplings in (5), (6), and (7) in the expressions for the slowroll formalism (3), (4), and (8) and the observables (11), (12), and (13) are identical to the classical case but with couplings that run with the RG scale. The cost is that canceling the Coleman-Weinberg part we have committed to a (time-dependent) choice of renormalization scale, and we may no longer vary it. We note that it is not generic, that the same choice of scale removes quantum corrections from both the field equation and Friedmann equation is not generic. We see an example below where it does not work out when we consider a two-field model of inflation.

In order to solve the RG equations, we still need to define the reference scale $\mu_{0}$, where the parameters have values $\lambda_{0}, m_{0}^{2}, \xi_{0}$. We choose to pick the scale $\mu_{0}$ to correspond to the initial value of $\phi, \phi_{0}$, deep in the slow-roll inflationary regime. Then, using the expression for $\mu$ defined above, we take

$$
\mu_{0}^{2}=\mu^{2}(0)=\frac{\left|M^{2}\left(\phi_{0}, H_{0}, \lambda_{0}, m_{0}^{2}, \xi_{0}\right)\right|}{e} .
$$

This is an explicit expression for $\mu_{0}$, if we use for $\phi_{0}$ the slow-roll approximation (10) to determine $H, \dot{\phi}$ and $\dot{H} \propto \epsilon_{1}=0$.

\section{SINGLE-FIELD NUMERICAL ANALYSIS}

\section{A. Classical evolution, minimally coupled}

In the classical limit, to get us started we can with relative ease solve the slow-roll equations semi-analytically, in either of the monomial limits $\left(m^{2}=0, \xi=0\right)$ or $(\lambda=0$, $\xi=0)$. Using the interval $N_{*}=50$ to 60 , we for each set of parameter values (either $m^{2}$ or $\lambda$ ) compute $n_{s}$ and $r$, imposing the central value of the Planck constraint on $A_{s}$ [33]

$$
\log \left(10^{10} A_{s}\right)=3.089 \pm 0.036
$$

This is shown as the two line segments in Fig. 1. We confirm the familiar result that classical $\phi^{4}$ inflation is firmly outside the observationally allowed region (shown as purple contours) and $\phi^{2}$ inflation marginally so. 


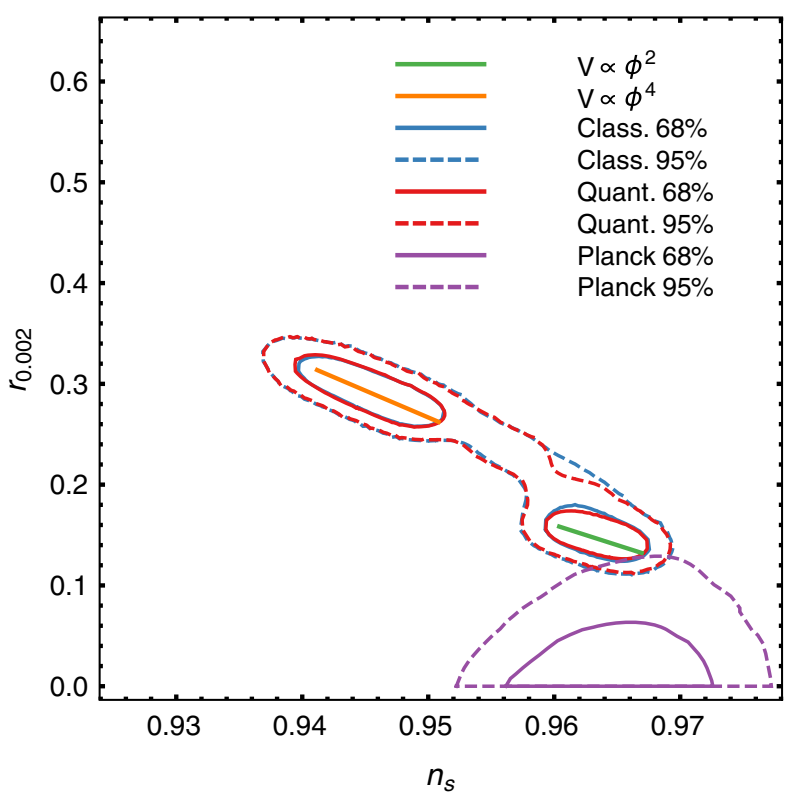

FIG. 1. The Planck-allowed region in the $n_{s}-r$ plane (purple). Overlaid, the slow-roll result for monomial $\phi^{2}$ (green) and $\phi^{4}$ (orange) inflation. Also, Non- slow-roll confidence intervals for the classical $\phi^{2}+\phi^{4}$ model (blue) and when quantum corrections are taken into account (red).

Overlaid are the results of a full numerical sampling, using classical dynamics where we allow to vary both $\lambda, m^{2}$ (so not restricting to monomial inflation) and the initial value $\phi_{0}$ in the intervals (see Sec. VI for details)

$$
\lambda_{0} \in\left[10^{-16} ; 10^{-10}\right], \quad \frac{m_{0}^{2}}{M_{\mathrm{pl}}^{2}} \in\left[10^{-16} ; 10^{-10}\right], \quad \frac{\phi_{0}}{M_{\mathrm{pl}}} \in[2 ; 30] .
$$

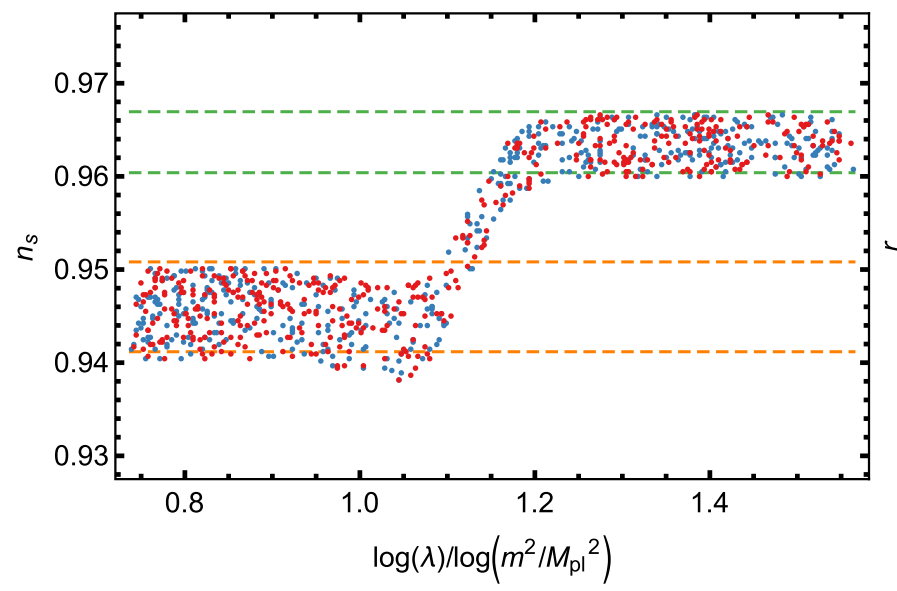

Note that, although we quote parameters with subscript 0 , in the classical simulation, these do not RG run and so are equal to $\lambda, m^{2}$ (and shortly also $\xi$ at all scales $\mu$ ). Here, $\phi_{0}$ is the initial field value throughout. Also, we do not employ the slow-roll approximation for the field or mode evolution. The slow-roll parameters only enter as we compute the observables to leading order in slow-roll [Eqs. (11), (12), (13)]. Rather than imposing the central value of $A_{s}$, we marginalize $A_{s}$ with a Gaussian distribution of width as in (26). These regions are bounded by red lines in Fig. 1.

We see that the allowed region in the $n_{s}-r$ is now an elongated "banana", nicely including the semi-analytic SR intervals inside the $68 \%$ confidence regions. This gives us confidence in the numerical implementation and also suggests that the slow-roll approximation is in fact rather good. With the given choice of the prior distributions for the parameters, observables close to the classical slow-roll results for monomial inflation are more likely than others. In Fig. 2, we see that statistically either the $\lambda$ or the $m^{2}$ term dominates the potential, and the solution of the field equation behaves accordingly.

\section{B. Quantum evolution, minimally coupled}

The red contours in Fig. 1 refer to a numerical scan of parameters $\xi_{0}, m_{0}^{2}, \lambda_{0}, \phi_{0}$ in the exact same ranges as before. But this time, the parameters run as the RG evolution, and scale $\mu$ changes in time as described above. We see that the classical and quantum regions in the $n_{s}-r$ plane are identical, up to statistical errors. We have checked that there is indeed convergence of the two regions as the statistics increases. In principle, one could imagine having large quantum corrections shifting individual parameter points around in the $n_{s}-r$ plane, which just happens to

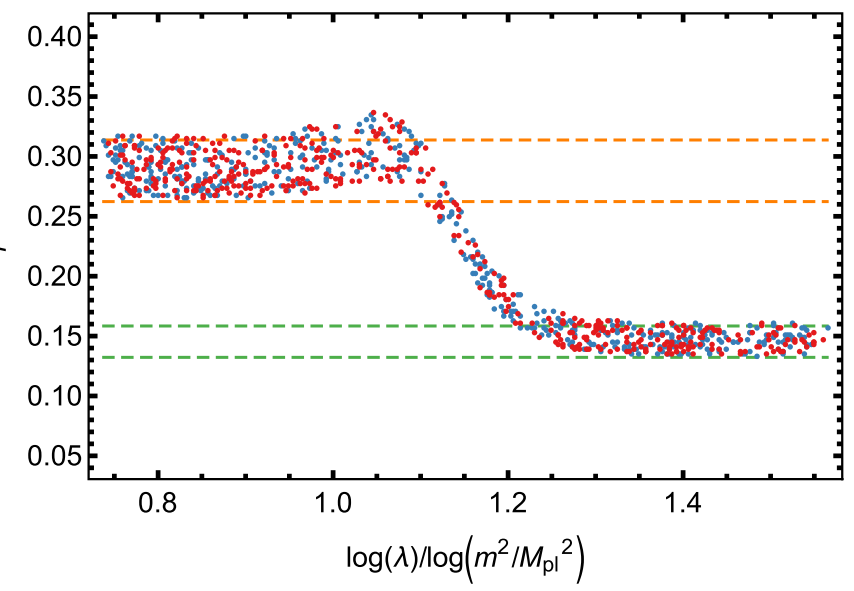

FIG. 2. Scatter plot showing the dependence of $n_{s}$ and $r$ on the ratio of the exponents of $\lambda$ and $m^{2}$. The dominating contribution to the potential typically determines the behavior of the model as "almost" $\phi^{2}$ or $\phi^{4}$ monomial inflation. The horizontal bands indicate the ranges of these models in classical slow-roll for $N_{*}$ between 50 and 60. Red and blue points represent data obtained with and without quantum corrections, respectively. Included are only data points whose value for $\log \left(10^{10} A_{s}\right)$ is within nine standard deviations of the Planck experimental value. 

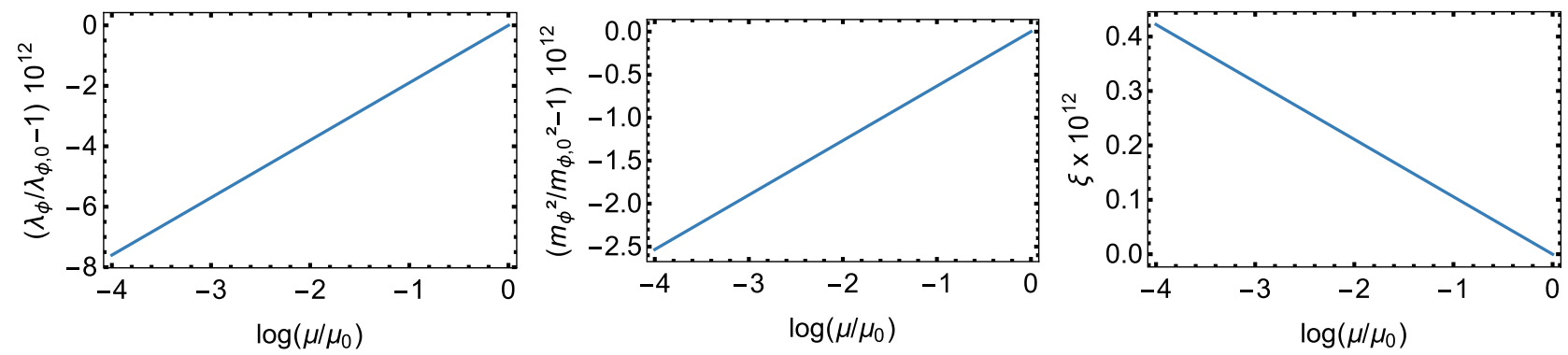

FIG. 3. The relative 1-loop running couplings for the one-field model. Left: $\lambda / \lambda_{0}-1$. Middle: $m^{2} / m_{0}^{2}-1$. Right: $\xi$. For all three parameters, we have multiplied by $10^{12}$. For illustration, we have used $\lambda_{0}=10^{-10}, m_{0}^{2}=10^{-10} M_{\mathrm{pl}}^{2}$, and $\xi_{0}=0$.

create the very same overall distribution. But we have checked that indeed for the individual points the correction is tiny.

To further investigate the quantum corrected behavior, we can analytically solve for the 1-loop running of the couplings as a function of scale $\mu$, finding

$$
\begin{aligned}
\lambda & =\frac{\lambda_{0}}{1-\frac{3 \lambda_{0}}{16 \pi^{2}} \log \left[\frac{\mu}{\mu_{0}}\right]}, \\
m^{2} & =m_{0}^{2} \frac{1}{\left(1-\frac{3 \lambda_{0}}{16 \pi^{2}} \log \left[\frac{\mu}{\mu_{0}}\right]\right)^{1 / 3}}, \\
\xi-\frac{1}{6} & =\left(\xi_{0}-\frac{1}{6}\right) \frac{1}{\left(1-\frac{3 \lambda_{0}}{16 \pi^{2}} \log \left[\frac{\mu}{\mu_{0}}\right]\right)^{1 / 3}} .
\end{aligned}
$$

An example is shown in Fig. 3, and we see that for the very small couplings required by observations the running is very small indeed, unless the scale changes by many orders of magnitude.

To further exemplify the magnitude of the running, let us simply solve for the field evolution in the classical slow-roll approximation, for the case $\xi=0, m^{2}=0$. Let us also, for the sake of argument, assume that the initial value of the field is $N_{e}=60 e$-folds before the end of inflation, the epoch of horizon crossing. Then, we have a change of scale during the whole evolution (initial value to end of inflation) of

$$
\begin{aligned}
\frac{\mu_{e}}{\mu_{0}} & =\left[\frac{\left|M^{2}\left(\phi_{e}, H_{e}\right)\right|}{\left|M^{2}\left(\phi_{H}, H_{H}\right)\right|}\right] \\
& =\left[\frac{\left|\frac{1}{2} \lambda \phi_{e}^{2} M_{\mathrm{pl}}^{2}+\frac{1}{12} \lambda\left(2-\epsilon_{1}^{e}\right) \phi_{e}^{4}\right|}{\left|\frac{1}{2} \lambda \phi_{H}^{2} M_{\mathrm{pl}}^{2}+\frac{1}{12} \lambda\left(2-\epsilon_{1}^{H}\right) \phi_{H}^{4}\right|}\right],
\end{aligned}
$$

where indices $e$ and $H$ denote the end of inflation and the horizon crossing epoch, respectively, and where we have used the relation

$$
R=H^{2}\left(12-6 \epsilon_{1}\right)=\frac{\lambda}{12} \phi^{4}\left(2-\epsilon_{1}\right) .
$$

Using $\epsilon_{1}=\phi^{2} /\left(8 M_{\mathrm{pl}}^{2}\right)$ for the classical $\lambda \phi^{4} / 24$ model under consideration, we find

$$
\frac{\mu_{e}}{\mu_{0}}=e^{-3.8} \simeq \frac{1}{45}, \quad \text { Monomial } \phi^{4} .
$$

For $\lambda_{0}=10^{-10}$, using $\mu=\mu_{0} / 45$, the combination $3 \lambda_{0} \log \left[\mu / \mu_{0}\right] /\left(16 \pi^{2}\right)$ featuring prominently in (28) is $\pm 10^{-11}$, making the running completely negligible. This conclusion does not change upon using 2-loop RG running, or considering a $m^{2} \phi^{2} / 2$ theory $(\lambda=0, \xi=0)$, where

$$
\frac{\mu_{e}}{\mu_{0}}=e^{-2.4} \simeq \frac{1}{11}, \quad \text { Monomial } \phi^{2} .
$$

Note that choosing the origin of the running $\mu_{0}$ to be much deeper in the slow-roll regime (i.e., much before the horizon crossing epoch) does not matter to the observables $n_{s}, r$, since they only depend on the value of the potential during and after the horizon crossing epoch. But it does change the mapping from observables to basic variables $\lambda_{0}, m_{0}^{2}$, since they will have run for a while before entering this epoch. Hence, only the running between $\phi_{H}$ and $\phi_{e}$ matters. As we see, this is very small, and for the one-field model, the running is the only quantum effect after choosing the pseudo-optimal RG scale $\mu(\phi, H, \ldots)$.

As a result, in a single-field inflation model, quantum corrections are very small. This follows from the running of the couplings, which are all controlled by $\lambda$ and the fact that (as we have seen) any other effect of quantum corrections can be made to disappear by a convenient choice of RG scale $\mu$. It is tempting to try to compensate the smallness of the coupling by making $\mu$ very small or large, so that the combination $\lambda \log \left(\mu / \mu_{0}\right)$ is much bigger. But that is precisely not allowed, since we have committed to a pseudo-optimal choice of $\mu$ in order to trust our perturbative approximation in the first place. We note that a further reduction of the $\mu$-dependence by finding the truly optimal expression for $\mu$ would only further emphasise this conclusion. We return to this point shortly.

We also note that although we are comparing the quantum evolution to a minimally coupled classical evolution, because $\xi$ runs with scale, this is not really well defined in the quantum case, except if setting the initial value to zero, $\xi_{0}=0$, as we do here. 


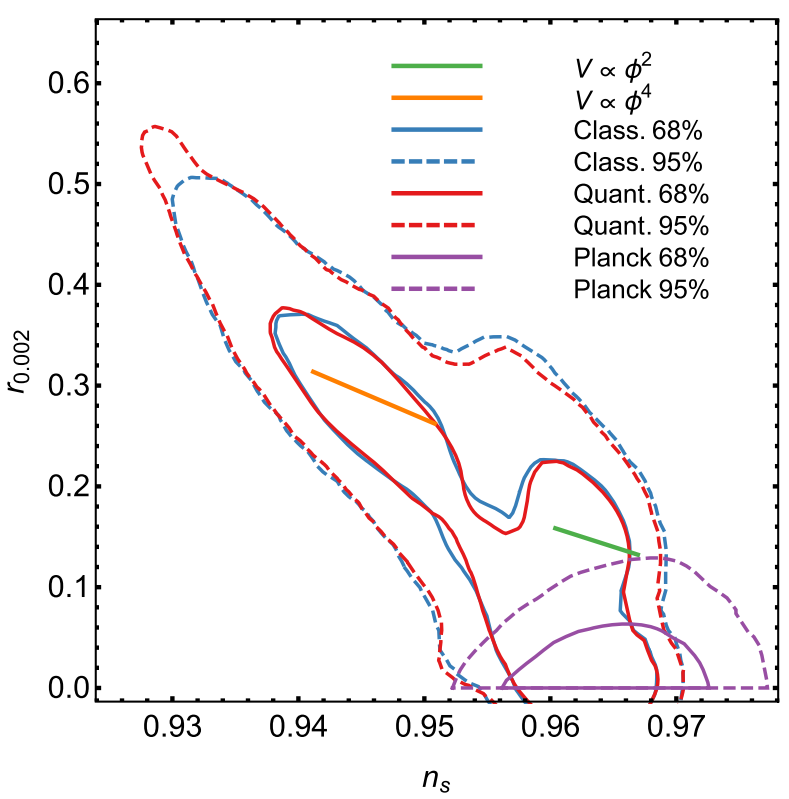

FIG. 4. The Planck-allowed region in the $n_{s}-r$ plane (purple). Overlaid, the slow-roll result for monomial $\phi^{2}$ (green) and $\phi^{4}$ (orange) inflation. In addition, confidence intervals for the $\phi^{2}+\phi^{4}+\xi R \phi^{2}$ model using classical dynamics (blue) and quantum corrected dynamics (red).

\section{Nonminimal coupling}

Allowing now for (initial) nonminimal couplings in the interval

$$
\xi_{0} \in\left[10^{-4} ; 1\right]
$$

we find Fig. 4. We perform the completely analogous numerical procedure as for the minimally coupled case, running scans with and without quantum corrections.

We again see a familiar result that allowing for nonminimal coupling the region consistent with the observed $A_{s}$ has a substantial overlap with the Planck-allowed region. There is still a weaker correlation between $n_{s}$ and $r$ along a diagonal in the plot. With our optimized choice of the RG scale, the quantum corrections do not significantly modify the posterior distribution for the observables. We found that allowing for nonzero initial $\xi$ destroys the simple relationship of Fig. 2: Any relative size of $\lambda$ and $m^{2}$ can be compensated for by a choice of $\xi$ to produce observables inside the Planck-allowed region. The probability distribution in fact takes its largest values close to $r=0$.

\section{Validating the choice of $\boldsymbol{\mu}$}

Because of the complicated dependence on dynamical variables $(\phi, \dot{\phi}, H, \dot{H})$, one cannot solve for the optimal $\mu$ in closed form

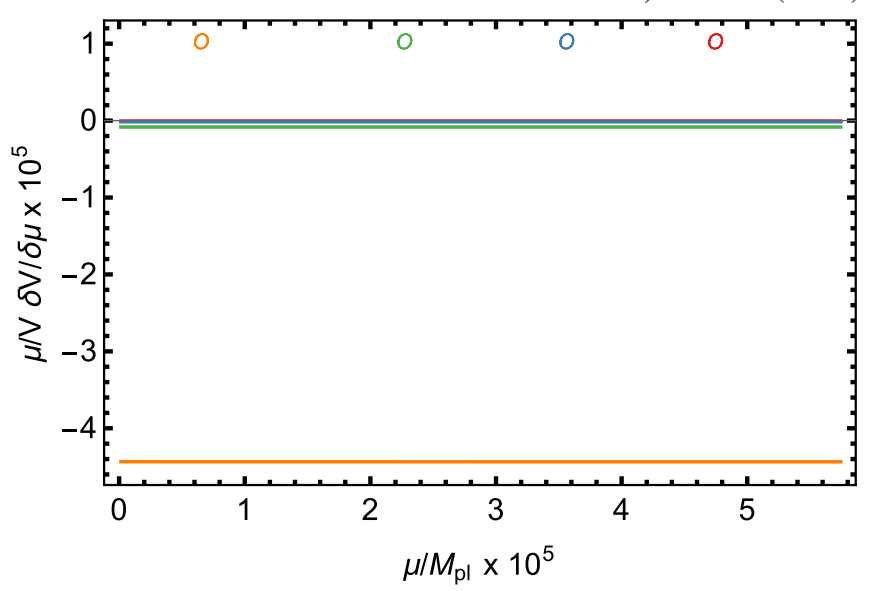

FIG. 5. The pseudo-optimal scale (circles) at different times (colors) and the relative dependence of the potential on $\mu$ given sets of $(\phi, \dot{\phi}, H, \dot{H})$ along the trajectory. Note the rescaling by $10^{5}$ along both axes.

$$
\frac{\mu}{V_{\mathrm{eff}}} \frac{d V_{\mathrm{eff}}}{d \mu}=0
$$

It is not even clear that such a minimum exists. Also, it makes little sense minimizing in the complete variables $+\mu$ space, since the dynamical variables are not independent but follow from a specific inflationary trajectory in field space. In Fig. 5, we show an example where we pick four times along a given trajectory in $(\phi, \dot{\phi}, H, \dot{H})$ space. For each of these, we compute (34) as a function of $\mu$ (dashed lines). We see that there is no obvious optimal value but that the dependence on $\mu$ is very weak at all times during the trajectory. The time-dependent value of the pseudo-optimal scale is indicated as circles. This scale moves towards smaller values during the evolution.

This completes our analysis of the single-field model.

\section{COUPLING TO A SPECTATOR FIELD}

For any realistic model of inflation, the inflaton field must be coupled to additional degrees of freedom and ultimately to the fields of the Standard Model. Because these have quantum fluctuations, their presence is encoded in the effective action of the inflaton itself. But now, because classically the coupling between the two is unconstrained by $\mathrm{CMB}$ observables, we can, in principle, imagine choosing it arbitrarily large, $\mathcal{O}(1)$. There are some constraints due to the reheating mechanism and how it affects the last few $e$-folds at the end of inflation. We do not take this complication into account here, since it requires to establish a full field theory simulation. But even for couplings much below unity, our expectation is that the allowed values of the parameters (say, $\lambda_{\phi}, m_{\phi}^{2}, \ldots$ ) are very much dependent on the inclusion of quantum corrections and the interactions with these other degrees of freedom. 
A simple example of this is to consider two scalar fields, the inflaton $\phi$ and a representative of all the other degrees of freedom $\sigma$. Note that $\sigma$ is not intended to be a curvaton. The $\mathrm{CMB}$ and the expansion of the Universe both originate from the dynamics of $\phi$. The action reads

$$
\begin{aligned}
S= & \int d^{n} x \sqrt{-g} \\
& \times\left[\frac{M_{\mathrm{pl}}^{2}}{2} R f(\phi, \sigma)+\frac{1}{2} \partial_{\mu} \phi \partial^{\mu} \phi-\frac{1}{2} m_{\phi}^{2} \phi^{2}-\frac{\lambda_{\phi}}{24} \phi^{4}\right. \\
& \left.-\frac{g}{4} \phi^{2} \sigma^{2}+\frac{1}{2} \partial_{\mu} \sigma \partial^{\mu} \sigma-\frac{1}{2} m_{\sigma}^{2} \sigma^{2}-\frac{\lambda_{\sigma}}{24} \sigma^{4}\right],
\end{aligned}
$$

with

$$
f(\phi, \sigma)=1-\frac{\xi_{\phi} \phi^{2}}{M_{\mathrm{pl}}^{2}}-\frac{\xi_{\sigma} \sigma^{2}}{M_{\mathrm{pl}}^{2}},
$$

and where for consistency we have again allowed for nonminimal coupling to gravity. We will stipulate that $\phi$ provides the dominant energy component through its mean field being displaced from the potential minimum, whereas $\sigma$ will be taken to be in its vacuum around $\sigma=0$. In this case, the classical two-field equations of motion are identical to those of the one-field model in the previous section, and the observables are computed in exactly the same way.

\section{A. Quantum corrections, two fields}

Computing the 1-loop effective potential, with 1-loop RG running, we have

$$
\begin{aligned}
V_{\text {eff }}= & \frac{1}{2} m_{\phi}^{2} \phi^{2}+\frac{1}{2} \xi_{\phi} R \phi^{2}+\frac{1}{24} \lambda_{\phi} \phi^{4} \\
& +\frac{1}{64 \pi^{2}} M_{\phi}^{4}(\phi)\left[\log \frac{\left|M_{\phi}^{2}(\phi)\right|}{\mu^{2}}-\frac{3}{2}\right] \\
& +\frac{1}{64 \pi^{2}} M_{\sigma}^{4}(\phi)\left[\log \frac{\left|M_{\sigma}^{2}(\phi)\right|}{\mu^{2}}-\frac{3}{2}\right],
\end{aligned}
$$

where as advertised we have already imposed that ${ }^{5} \sigma=0$. But, contrary to the classical limit, at the quantum level the presence of the spectator field $\sigma$ is apparent in the logarithmic corrections to the effective potential. We now have two effective mode masses,

$$
M_{\phi}^{2}(\phi)=m_{\phi}^{2}+\frac{\lambda_{\phi}}{2} \phi^{2}+\left(\xi_{\phi}-1 / 6\right) R
$$

\footnotetext{
${ }^{5}$ Here, $\sigma=0$ is a solution also of the quantum evolution, had we written down and solved for the $\sigma$ equation of motion.
}

$$
M_{\sigma}^{2}(\phi)=m_{\sigma}^{2}+\frac{g}{2} \phi^{2}+\left(\xi_{\sigma}-1 / 6\right) R \text {. }
$$

We expect that $\lambda_{\phi}$ is small to allow for slow-roll inflation. On the other hand, $g$ need not be, and so generically $M_{\sigma}^{2} \simeq g \phi^{2} / 2$, much larger than the Hubble rate $H^{2}$. Hence for the purposes of CMB observables, $\sigma$ is a heavy field and does not contribute to the density perturbations. This is as expected and a useful simplification. The equation of motion for $\phi$ follows by variation of the effective action,

$$
\begin{aligned}
\ddot{\phi}+ & 3 H \dot{\phi}+\left(m_{\phi}^{2}+\xi_{\phi} R\right) \phi+\frac{1}{6} \lambda_{\phi} \phi^{3} \\
& +\frac{\lambda_{\phi} \phi M_{\phi}^{2}(\phi)}{32 \pi^{2}}\left(\log \frac{\left|M_{\phi}^{2}(\phi)\right|}{\mu^{2}}-1\right) \\
& +\frac{g \phi M_{\sigma}^{2}(\phi)}{32 \pi^{2}}\left(\log \frac{\left|M_{\sigma}^{2}(\phi)\right|}{\mu^{2}}-1\right)=0 .
\end{aligned}
$$

We can now choose a pseudo-optimal RG scale $\mu$ in such a way that the two logarithmic terms cancel out. We have

$\frac{\mu^{2}}{M_{\mathrm{pl}}^{2}}=e^{-1} \exp \left(\frac{\lambda_{\phi} M_{\phi}^{2}(\phi) \log \left[\frac{\left[M_{\phi}^{2}(\phi) \mid\right.}{M_{\mathrm{pl}}^{2}}\right]+g M_{\sigma}^{2} \log \left[\frac{\left[M_{\sigma}^{2}(\phi) \mid\right.}{M_{\mathrm{pl}}^{2}}\right]}{\lambda_{\phi} M_{\phi}^{2}(\phi)+g M_{\sigma}^{2}(\phi)}\right)$.

Again, this is not the truly optimal choice but is a very convenient one. This also trivially means that

$$
\left(\log \frac{\left|M_{\sigma}^{2}(\phi)\right|}{\mu^{2}}-1\right)=-\frac{\lambda_{\phi} M_{\phi}^{2}(\phi)}{g M_{\sigma}^{2}(\phi)}\left(\log \frac{\left|M_{\phi}^{2}(\phi)\right|}{\mu^{2}}-1\right) .
$$

As for the single-field case, the Friedmann equations do not follow from variation of the effective action but from a separate computation, varying the classical action with respect to $g^{\mu \nu}$. There are now two fields, each with their contribution (even after setting $\sigma=0$ ). We have

$$
\begin{aligned}
T_{\mu \nu}^{Q}= & -g_{\mu \nu} \frac{H^{4}}{64 \pi^{2}}\left[A_{\phi}\left(\log \frac{\left|M_{\phi}^{2}(\phi)\right|}{\mu^{2}}-1\right)\right. \\
& \left.+A_{\sigma}\left(\log \frac{\left|M_{\sigma}^{2}(\phi)\right|}{\mu^{2}}-1\right)\right],
\end{aligned}
$$

with

$$
\begin{aligned}
A_{\phi}= & {\left[\delta_{\phi}^{2}-4 \delta_{\phi} \epsilon_{1}-2 \delta_{\phi}-6 \epsilon_{1}\right.} \\
& \left.+12 \xi_{\phi}\left(2-\delta_{\phi}+\epsilon_{1}-\delta_{\phi} \epsilon_{1}\right)\right], \\
A_{\sigma}= & {\left[\delta_{\sigma}^{2}-4 \delta_{\sigma} \epsilon_{1}-2 \delta_{\sigma}-6 \epsilon_{1}\right.} \\
& \left.+12 \xi_{\sigma}\left(2-\delta_{\sigma}+\epsilon_{1}-\delta_{\sigma} \epsilon_{1}\right)\right] .
\end{aligned}
$$


The $\epsilon_{1}$ is defined as before, and we again have the slowroll-like quantities

$\delta_{\phi}=\frac{m_{\phi}^{2}+\frac{\lambda_{\phi}}{2} \phi^{2}+\xi_{\phi} R}{H^{2}}, \quad \delta_{\sigma}=\frac{m_{\sigma}^{2}+\frac{g}{2} \phi^{2}+\xi_{\sigma} R}{H^{2}}$.

Note however that $\delta_{\sigma}$ is not small in the slow-roll sense. Here, $\sigma$ is not a light field. It is clear that the choice of $\mu$, Eq. (42), no longer makes the quantum corrections vanish in the expression for the energy density (as they did for the one-field case). However, it does allow us to replace one logarithm by another, in accordance with (43), to get

$$
\begin{aligned}
T_{\mu \nu}^{Q}= & -g_{\mu \nu} \frac{H^{4}}{64 \pi^{2}}\left(\log \frac{\left|M_{\phi}^{2}(\phi)\right|}{\mu^{2}}-1\right) \\
& \times\left(A_{\phi}-A_{\sigma} \frac{\lambda_{\phi} M_{\phi}^{2}(\phi)}{g M_{\sigma}^{2}(\phi)}\right) .
\end{aligned}
$$

It is worth looking at the size of this object, in the simplified case of $\xi_{\phi}=\xi_{\sigma}=m_{\sigma}=\lambda_{\sigma}=0$ (also neglecting, for now, their reappearance due to running). Then, neglecting all SR-sized quantities, we have

$$
\begin{aligned}
T_{\mu \nu}^{Q} \simeq & -g_{\mu \nu} \frac{H^{4}}{64 \pi^{2}}\left(\log \frac{\left|M_{\phi}^{2}(\phi)\right|}{\mu^{2}}-1\right) \\
& \times\left\{-\frac{g^{2} \phi^{4}}{4 H^{4}}+2 \frac{g \phi^{2}}{2 H^{2}}\right\} \frac{\lambda_{\phi}\left(m_{\phi}^{2}+\frac{\lambda_{\phi}}{2} \phi^{2}\right)}{\frac{g \phi^{2}}{2}}, \\
\simeq & g_{\mu \nu} \frac{\lambda_{\phi} g}{64 \pi^{2}}\left(\frac{m_{\phi}^{2}}{2} \phi^{2}+\frac{\lambda_{\phi}}{4} \phi^{4}\right)\left(\log \frac{\left|M_{\phi}^{2}(\phi)\right|}{\mu^{2}}-1\right) .
\end{aligned}
$$

This may be compared to the tree-level contributions

$$
\simeq g_{\mu \nu}\left(\frac{m_{\phi}^{2}}{2} \phi^{2}+\frac{\lambda_{\phi}}{24} \phi^{4}\right),
$$

which are seen to dominate as long as

$$
\frac{g \lambda_{\phi}}{128 \pi^{2}}\left(\log \frac{\left|M_{\phi}^{2}(\phi)\right|}{\mu^{2}}-1\right) \ll \frac{1}{2} \quad \text { or } \quad \frac{1}{12} .
$$

This can easily be accommodated. We emphasise that this criterion follows from the choice of RG scale $\mu$, since without the substitution of (43) the prefactor of the logarithm would have been $\left(g \phi^{2}\right)^{2}$, which would dominate the tree-level contribution as soon as $g^{2} \simeq 100 \lambda_{\phi}$. This in turn would be possible for quite sensible values of $g$. So, although the RG scale does not allow us to cancel out the quantum corrections completely in this case, it does suppress them to a subleading contribution, compared to contributions at tree level. We confirm this a posteriori below, and it means that we can again identify the leading quantum corrections to be the RG running of the couplings.

\section{B. RG running for two fields}

There are now seven RG running couplings $m_{\phi}^{2}, m_{\sigma}^{2}, \lambda_{\phi}, \lambda_{\sigma}, \xi_{\phi}, \xi_{\sigma}, g$, and we derive a set of coupled 1-loop RG equations,

$$
\begin{aligned}
\frac{1}{\mu} \frac{d \lambda_{\phi}}{d \mu} & =\frac{3}{(4 \pi)^{2}}\left[\lambda_{\phi}^{2}+g^{2}\right], \\
\frac{1}{\mu} \frac{d \lambda_{\sigma}}{d \mu} & =\frac{3}{(4 \pi)^{2}}\left[\lambda_{\sigma}^{2}+g^{2}\right], \\
\frac{1}{\mu} \frac{d m_{\phi}^{2}}{d \mu} & =\frac{1}{(4 \pi)^{2}}\left[\lambda_{\phi} m_{\phi}^{2}+g m_{\sigma}^{2}\right], \\
\frac{1}{\mu} \frac{d m_{\sigma}^{2}}{d \mu} & =\frac{1}{(4 \pi)^{2}}\left[\lambda_{\sigma} m_{\sigma}^{2}+g m_{\phi}^{2}\right], \\
\frac{1}{\mu} \frac{d \xi_{\phi}}{d \mu} & =\frac{1}{(4 \pi)^{2}}\left[\lambda_{\phi}\left(\xi_{\phi}-1 / 6\right)+g\left(\xi_{\sigma}-1 / 6\right)\right], \\
\frac{1}{\mu} \frac{d \xi_{\sigma}}{d \mu} & =\frac{1}{(4 \pi)^{2}}\left[\lambda_{\sigma}\left(\xi_{\sigma}-1 / 6\right)+g\left(\xi_{\phi}-1 / 6\right)\right], \\
\frac{1}{\mu} \frac{d g}{d \mu} & =\frac{1}{(4 \pi)^{2}} g\left[\lambda_{\phi}+\lambda_{\sigma}\right] .
\end{aligned}
$$

We are mostly interested in the dependence on the coupling $g$, but we see that $\lambda_{\phi}, \lambda_{\sigma}$, and $g$ source each others RG running, and so if $g$ is nonzero, they all are. Similarly, only if both masses $m_{\phi}$ and $m_{\sigma}$ vanish, do they remain zero. Finally, if both $\xi_{\phi}$ and $\xi_{\sigma}$ are equal to their conformal value $1 / 6$, they do not run. But if either is nonconformal (for instance, zero), they both run away from their initial value.

The central observation is that even if $\lambda_{\phi}$ starts out with a value of $\simeq 10^{(-10,-11,-12)}$, in accordance with the classical observational constraints, it grows semilinearly as $3 /(4 \pi)^{2} g^{2} \times \log \left[\mu / \mu_{0}\right]$. Hence even for a reasonable, perturbative value of $g=10^{-4}, \lambda_{\phi}$ will have grown to $>10^{-10}$ with a scale change of $\log \left[\mu / \mu_{0}\right]=\mathcal{O}(1)$. This would naively suggest that the system is driven out of slow-roll. It is therefore highly conceivable that the inclusion of quantum fluctuations puts strong constraints on the viable values of $g$, and at the same time, a careful choice of $g$ may allow otherwise ruled out scenarios (such as quartic inflation) to fit observations. We investigate this further below. In Fig. 6, we show one example of the running couplings, using $m_{\phi, 0}^{2}=10^{-10} M_{\mathrm{pl}}^{2}, \lambda_{\phi, 0}=10^{-10}$, and $g_{0}=10^{-4}$.

We observe that all parameters become nonzero. The left panel shows the relative change of the inflaton selfcoupling. We see that it can grow by a factor of 1-10 within the expected change of scale $\log \left[\mu / \mu_{0}\right]=-4$. In this example, we have used the maximum value of this initial coupling and the largest value of $g_{0}$, suggesting that quantum corrections may become important but need not be. We see shortly what the effect is.

We note in passing that the nonminimal coupling remains very small and that the relative change in the inflaton mass parameter $m_{\phi}^{2}$ is very small. Hence, the 

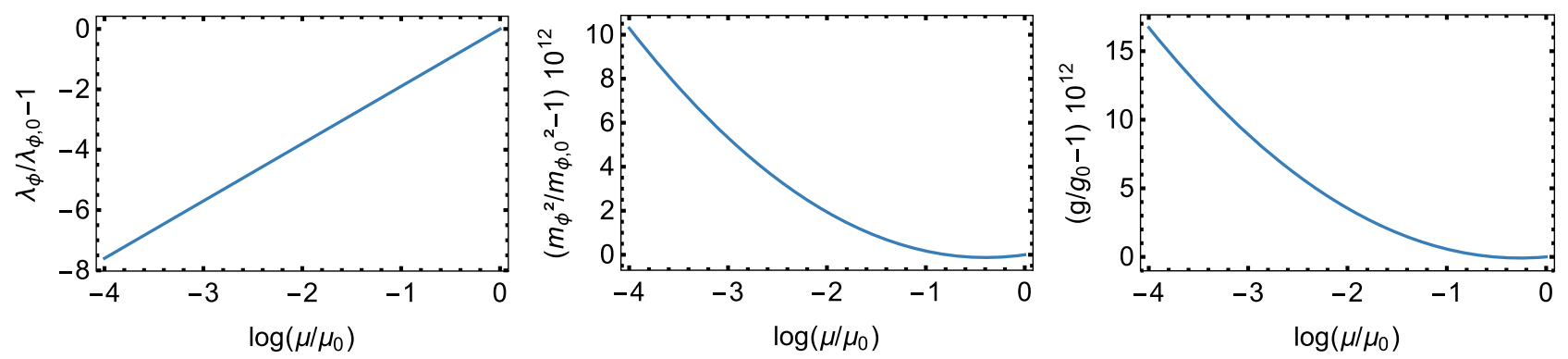

FIG. 6. The 1-loop running couplings for the two-field model. While $m_{\phi}^{2}$ and $g$ are again multiplied by $10^{12}$, the relative running of $\lambda_{\phi}$ is not. For illustration, we have used $\lambda_{\phi, 0}=10^{-10}, m_{\phi, 0}^{2}=10^{-10} M_{\mathrm{pl}}^{2}$, and $g_{0}=10^{-4}$. The remaining parameters $\lambda_{\sigma}, m_{\sigma}^{2}, \xi_{\phi, \sigma}$ also run (not shown).

primary effect of the RG running is for the inflaton selfcoupling to change significantly during the time evolution. The inflaton self-coupling $\lambda_{\sigma}$ grows as large as $\lambda_{\phi}$ but does not enter in the expressions for the observables.

\section{TWO-FIELD NUMERICAL ANALYSIS}

\section{A. Individual trajectories}

To illustrate the impact of quantum effects on the cosmological evolution, we first choose three examples for which inflation is successful. The initial parameters at $\mu=\mu_{0}$ for these cases are

$$
\begin{array}{rlrl}
\text { BM 1 : } m_{\phi, 0}^{2} & =6.2 \times 10^{-11} M_{\mathrm{pl}}^{2}, & \lambda_{\phi, 0}=5.9 \times 10^{-14}, \\
g_{0} & =6.4 \times 10^{-6}, \quad \phi_{0}=25 M_{\mathrm{pl}}, \\
\text { BM 2: } m_{\phi, 0}^{2} & =1.5 \times 10^{-12} M_{\mathrm{pl}}^{2}, & \lambda_{\phi, 0}=2.7 \times 10^{-16}, \\
g_{0} & =2.3 \times 10^{-10}, & \phi_{0}=21 M_{\mathrm{pl}}, \\
\text { BM 3: } m_{\phi, 0}^{2} & =1.5 \times 10^{-13} M_{\mathrm{pl}}^{2}, \quad \lambda_{\phi, 0}=4.6 \times 10^{-15}, \\
g_{0} & =2.2 \times 10^{-7}, \quad \phi_{0}=30 M_{\mathrm{pl}},
\end{array}
$$

and $\xi_{\phi, 0}=\lambda_{\sigma, 0}=\xi_{\sigma, 0}=0$ for BM 1-BM 3. We choose $N_{*}=55$ as the pivot scale everywhere and $\epsilon_{1}=1$ as the condition for the end of inflation.
We implement the numerics as for the one-field model. The dynamical variables are the same, but the evolution equations and solving for the time-dependent $\mu$ are more involved. The classical limit is identical to the one-field case (since we are postulating that the second field does not contribute to the energy density of the Universe nor to the density perturbations).

For the three parameter sets, we obtain the following numerical values for the observables:

$$
\begin{aligned}
\text { BM 1: } A_{s} & =3.0 \times 10^{-9}, \quad \delta A_{s}=-0.046 . \\
n_{s} & =0.96, \quad \delta n_{s}=0.0013 . \\
r & =0.14, \quad \delta r=-0.052 . \\
\text { BM 2: } A_{s} & =7.7 \times 10^{-11}, \quad \delta A_{s}=-3.1 \times 10^{-9} . \\
n_{s} & =0.96, \quad \delta n_{s}=5.6 \times 10^{-11} . \\
r & =0.15, \quad \delta r=1.0 \times 10^{-9} . \\
\text { BM 3: } A_{s} & =9.3 \times 10^{-12}, \quad \delta A_{s}=-0.065 . \\
n_{s} & =0.96, \quad \delta n_{s}=0.00059 . \\
r & =0.24, \quad \delta r=0.0056 .
\end{aligned}
$$

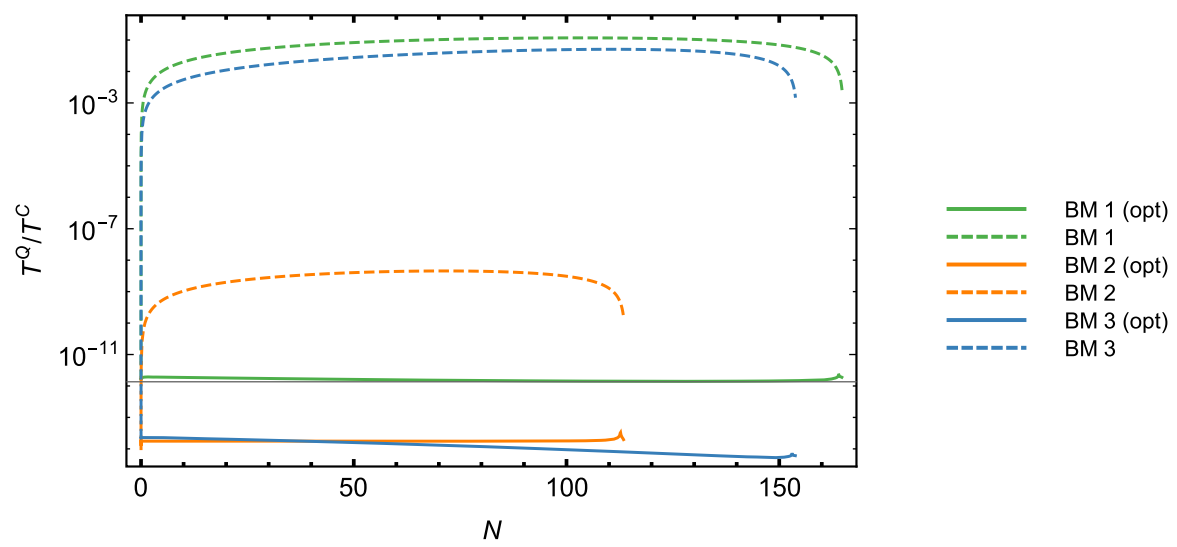

FIG. 7. Relative size of the quantum corrections to the Friedmann equations as a function of the evolved number of $e$-folds. The dashed lines correspond to these contributions with constant RG scale, while the full lines show their size if the RG scale is updated (in both cases computed along the trajectory obtained with only RG running corrections). 

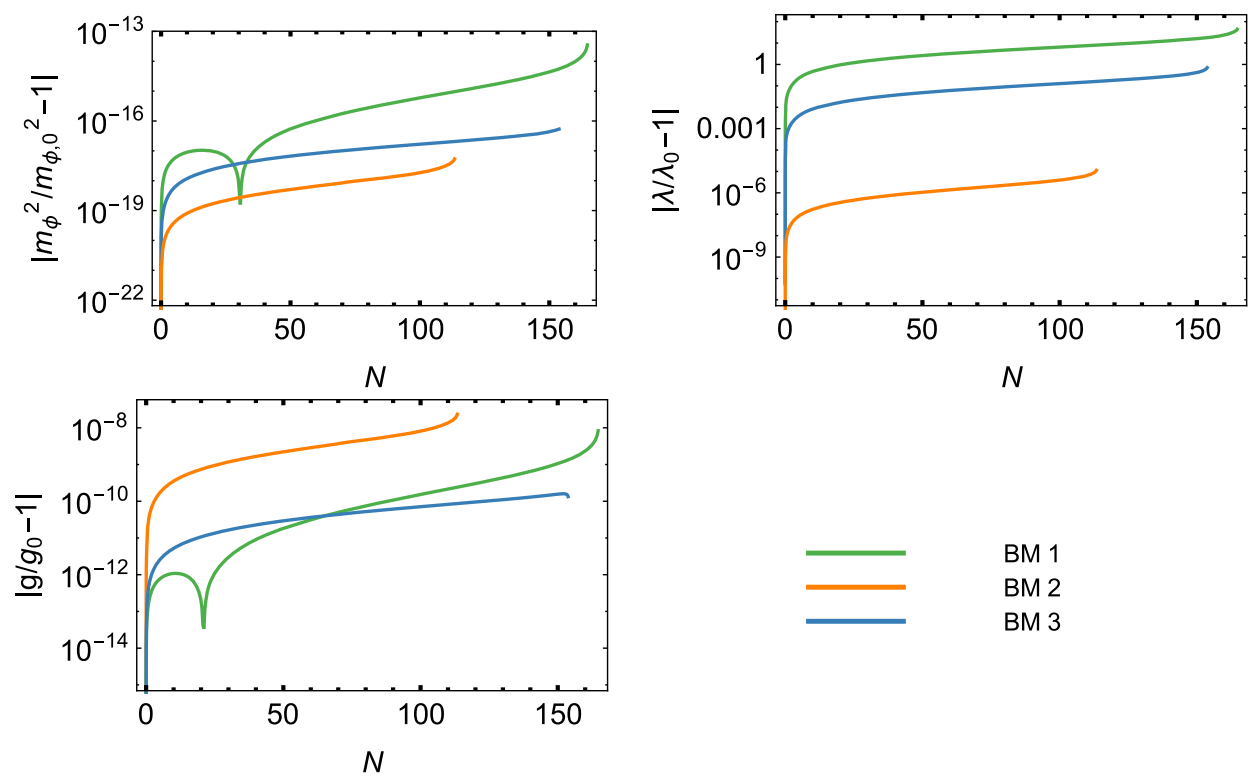

BM 1

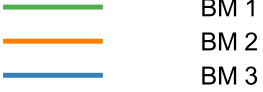

FIG. 8. Relative change of some of the two-field model parameters as a function of the evolved number of $e$-folds $N$ for different initial parameters (BM 1-BM 3). Further parameters that have zero initial values are not shown but run as well.

Here, $\delta A_{s}, \delta n_{s}$ and $\delta r$ represent the relative changes compared to the classical results. We see that the largest differences in these examples are at the percent level.

As described, we update the renormalization scale to remove the quantum contributions to the field equation. We find a posteriori that the remnant corrections to the Friedmann equations are negligible with this choice of $\mu$ (see Fig. 7). We show the magnitude along BM 1-BM 3 of the logarithmic corrections to the Friedmann equations, relative to the nonlogarithmic contributions. When
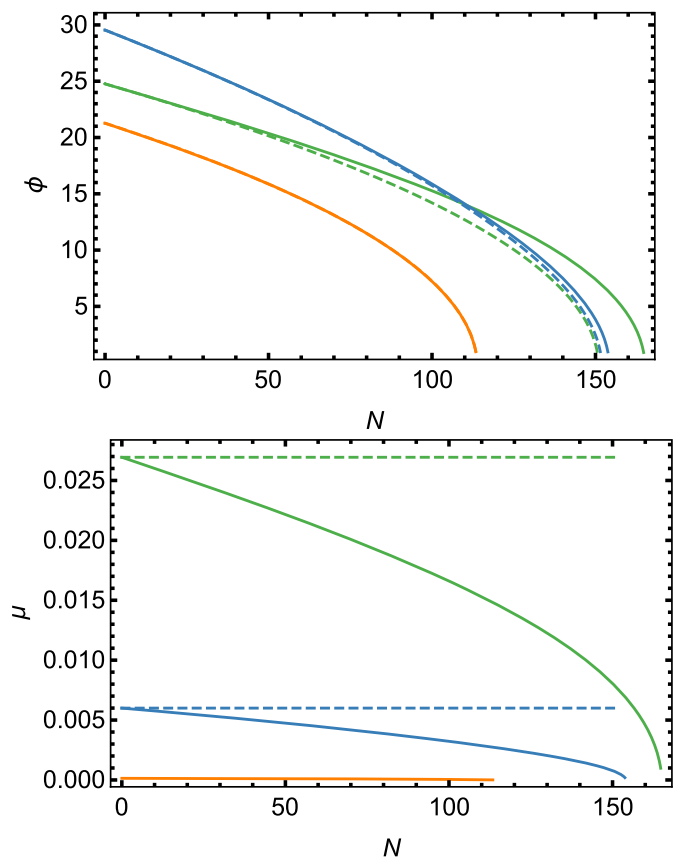

the RG scale is not adjusted over time as (42) (dashed lines), the contributions are small but may still be a few percent or more. By adjusting the RG scale (full lines), we can reduce this to one part in $10^{-11}$ or less. So although we are not able to identically cancel out the logarithms as for the one-field model, choosing the RG scale wisely is a vast improvement on even the "best" constant RG scale. We therefore ignore these contributions keeping only the $\mathrm{RG}$ running of the couplings according to Eqs. (52).
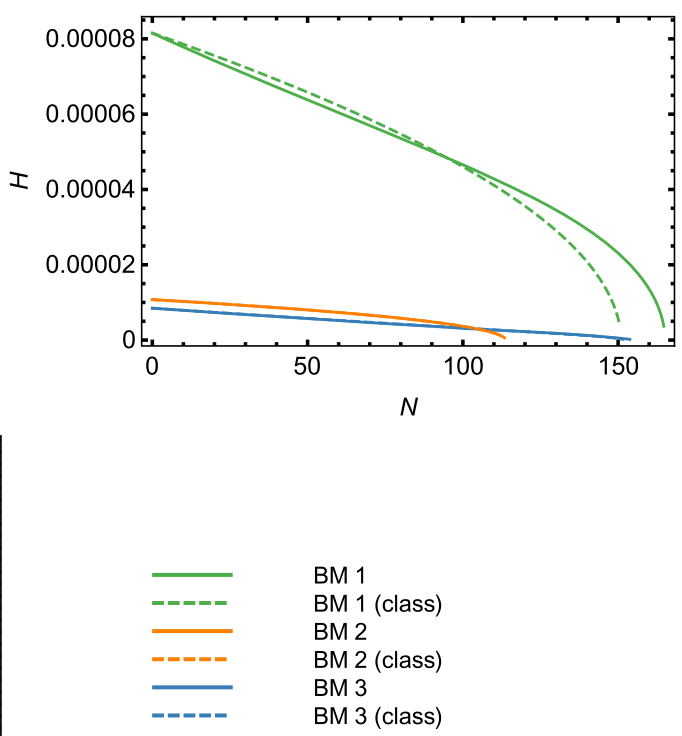

FIG. 9. Evolution of field value, Hubble rate, and the inferred renormalization scale. For large values of $g$ (BM 1), the RG running of the parameters can have a noticeable effect on the cosmological evolution. 


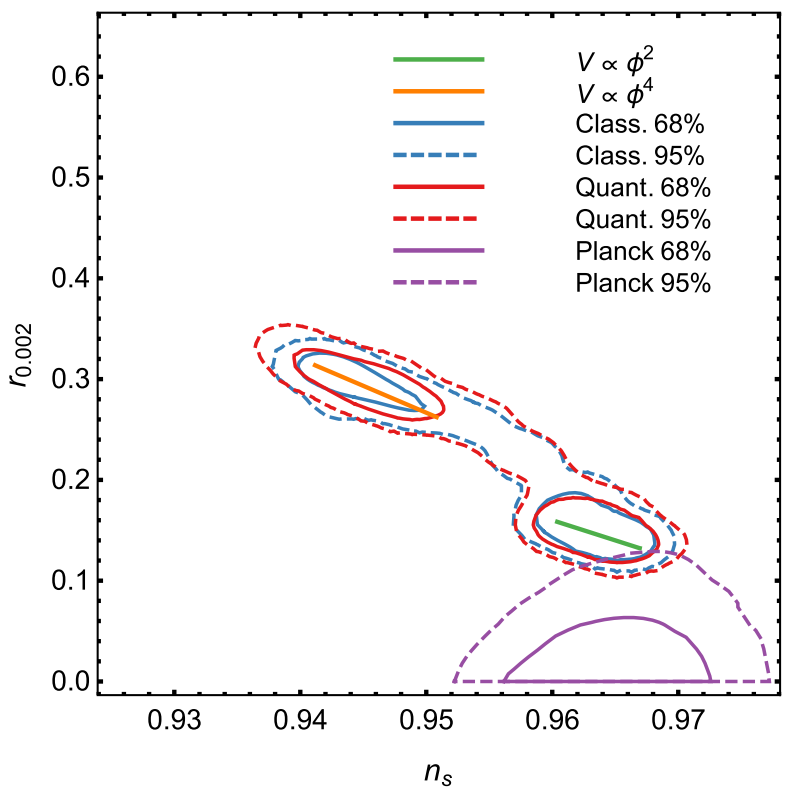

FIG. 10. The confidence regions for classical and quantum evolution the two-field case in the $n_{s}-r$ plane. Overlaid the Planck- allowed range and the slow-roll monomial benchmarks (orange and green lines).

The relative change of $m_{\phi}^{2}, \lambda_{\phi}$, and $g$ due to RG running is shown in Fig. 8. For larger values of $g$, this change can be noticeable. They are reflected in the quantum evolution of the field shown in Fig. 9.

\section{B. Full parameter scan}

It is tempting to search for parameter sets that maximize the effect of the quantum contributions. However, we want to convey an unbiased picture of their relevance. As before, we therefore sample the model parameters and initial conditions and evolve the non-slow roll equations of motion, computing the basic CMB observables. Marginalizing over $A_{s}$, we again generate a region in the $n_{s}-r$ plane. To reduce the sampling parameter space somewhat, we take $\lambda_{\sigma, 0}=0, m_{\sigma, 0}^{2}=0, \xi_{\phi, 0}=0$, and $\xi_{\sigma, 0}=0$ throughout. Because they all run, they only vanish at $\phi_{0}$.
This leaves as initial input $\lambda_{\phi, 0}, m_{\phi, 0}^{2}$, and the coupling $g_{0}$. We again take the ranges (27) and add

$$
g_{0} \in\left[10^{-16} ; 10^{-4}\right] .
$$

Figure 10 shows the two-field classical and quantum regions consistent with observations in the $n_{s}-r$ plane. As before, the slow-roll monomial results are indicated by the orange and green line segments, and the Planck results are marked with purple lines. For the present sweep, $\xi_{\phi, 0}$ is zero. As a consequence, the classical result is the "banana"-shaped $\phi^{2}+\phi^{4}$ region from Fig. 1 . Without the RG running, this agreement with the classical limit of the single-field model should be exact, and if $g$ is small, it should hold approximately, since the quantum contributions by the other nonvanishing parameters are tiny.

We see that the overall differences between the evolution with both small $g$ and large $g$ are small. This is somewhat surprising, since we have seen that at least for trajectories of the BM 1 type both evolution and observables are shifted by some percent. Apparently, when sampling over the whole range, one recovers the same allowed region, even though individual trajectories (corresponding to a given parameter set) are moved around within this region.

One may speculate that even larger values of $g$ will lead to larger effects such that the $n_{s}-r$ contour is brought into overlap with the Planck-allowed region. However, we find that for $g \gtrsim 10^{-5}$, the observed value for $A_{s}$ is not met any more (see Fig. 11), and as $\gtrsim 10^{-4}$, inflation is ruined altogether by the running of $\lambda_{\phi}$ sourced by large $g$. It is possible that carefully tuning to the narrow region around $g \simeq 10^{-5}$ one may find some singular cases with large corrections to the observables, but by a flat-logarithmic sampling as we do here, such a region does not show up.

\section{Validating the choice of $\boldsymbol{\mu}$}

Along a trajectory in $(\phi, \dot{\phi}, H, \dot{H})$ space, we again compute the relative dependence on the scale (34). In Fig. 12 is then the analogue of Fig. 5 but for two coupled
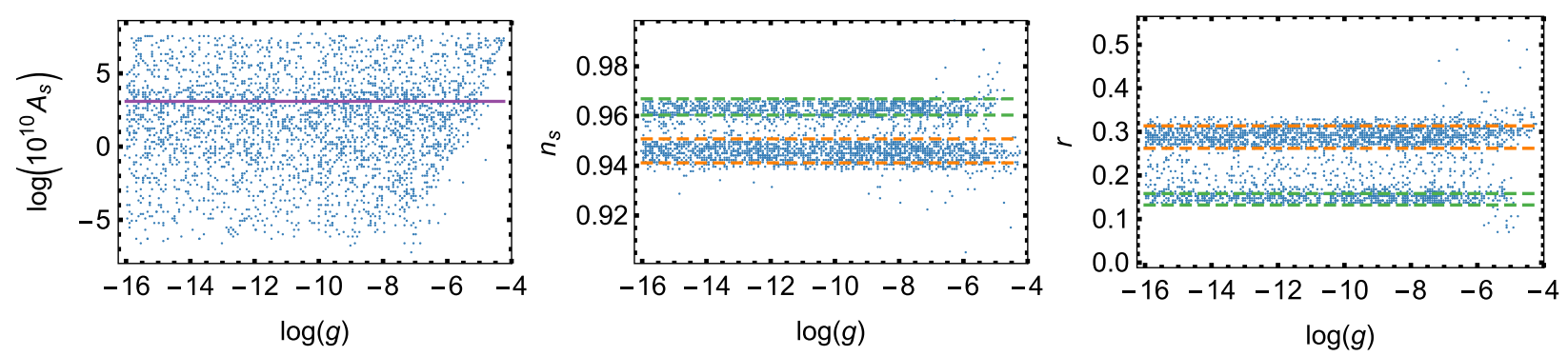

FIG. 11. Scatter plots showing the observables as a function of $g$. For large values, we see a deviation from the region given by the slow-roll results for monomial inflation. However, for $g \gtrsim 10^{-5}$, the data fails to satisfy the experimental constraints on $A_{s}$ indicated by the solid purple horizontal line. For $\gtrsim 10^{-4}$, inflationary evolution does not occur at all. 


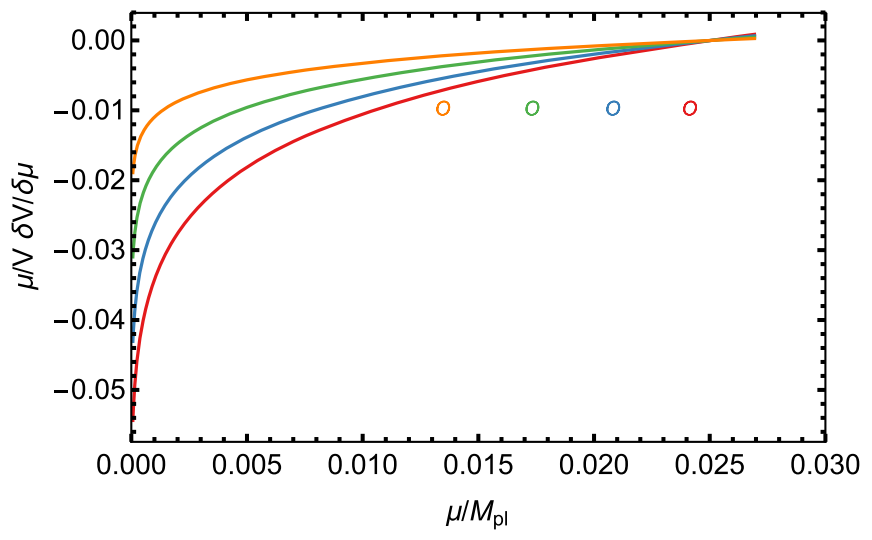

FIG. 12. The pseudo-optimal scale at different times (circles) and the potential as a function of $\mu$ given sets of $(\phi, H, \dot{H})$ along the trajectory.

fields. We see that the dependence on $\mu$ is much larger than for the one-field model (Fig. 5 was scaled up with $10^{5}$, Fig. 12 is not) but still at the percent level for most of the range shown. Our time-dependent, pseudo-optimal choice of scale (circles) nicely traverses the range of scales, never coming near the singular region near the $\mathrm{x}$-axis. In fact, we see that as the pseudo-optimal scale decreases the correspondingly color-coded $\mu$ dependence of $V_{\text {eff }}$ (red to orange) becomes more shallow. We have not observed a trajectory where the pseudo-optimal scale has caught up with the large $\mu$-dependence region. We conclude that our prescription for the choice of scale, apart from being very convenient, also ensures small $\mu$ dependence.

\section{NUMERICAL IMPLEMENTATION}

In this section, we provide some more details on how we solve the field and Friedmann equations and how we sample the parameters. Our numerical implementation is based on the public Fortran package Multimodecode [26]. It is suited to the computation of observables for multifield inflation models in the $\delta N$ formalism or by solving the mode equations directly. In the present work, we make use of the former feature only.

We however have to adapt the code significantly in order to support the nonminimal coupling to gravity as well as the quantum-corrected field (16) and Friedmann equations (17) [or Eqs. (41) and (44) for the two-field model] that take a nonstandard form. They represent a coupled system of ordinary differential and algebraic (constraint) equations. This system determines the evolution of mean field values and the Hubble rate in time or, after a corresponding transformation, in the number of $e$-folds since the beginning of inflation. Usually, the algebraic first Friedmann equation is used to explicitly solve for $H$.

With quantum corrections, however, its right-hand side depends in a complicated way on $H$. This is true even if the logarithms in (16) and (17) are eliminated by choice of the renormalization scale, because $\mu$ then becomes a function of $H$ (as well as $\phi$ and $\dot{\phi}$ ). Recall that, in each step, $\mu$ is determined as the solution of the algebraic equation (25) for $\mu$.

To avoid numerically solving the Friedmann equation, which would also need to be repeated in each time step of the evolution, we choose to solve the second Friedmann equation for $d H / d N$. In the single-field case, this is possible explicitly if the quantum terms are made zero through the choice of $\mu$ described above. In the two-field case, the choice of $\mu$ that eliminates the direct quantum contributions to the mean field equation leaves a nonvanishing quantum contribution to both Friedmann equations. As argued above, for the purposes of the present paper, we can neglect these terms in numerical computations.

For both models, we then arrive at the first order system (with $m_{\phi}^{2}=m^{2}, \lambda_{\phi}=\lambda$ and $\xi_{\phi}=\xi$ in the one-field case)

$$
\begin{aligned}
\phi^{\prime}= & v \\
v^{\prime}= & \frac{H^{\prime} v}{H} \\
& -\frac{1}{H^{2}}\left(3 H^{2} v+\left(m_{\phi}^{2}+\xi_{\phi} 6 H\left(H^{\prime}+2 H\right)\right) \phi+\frac{1}{6} \lambda_{\phi} \phi^{3}\right), \\
H^{\prime}= & \frac{H^{-1}}{48\left(\xi_{\phi}\left(6 \xi_{\phi}-1\right) \phi^{2}+1\right)} \\
& \times\left[1 2 H ^ { 2 } \left(6 \xi_{\phi}\left(1-8 \xi_{\phi}\right) \phi^{2}\right.\right. \\
& \left.-4 \xi_{\phi} \phi v+\left(4 \xi_{\phi}-1\right) \phi^{2}-6\right) \\
& \left.+\phi^{2}\left(\lambda_{\phi}\left(1-8 \xi_{\phi}\right) \phi^{2}+m_{\phi}^{2}\left(12-48 \xi_{\phi}\right)\right)\right],
\end{aligned}
$$

where the primes denote the derivative with respect to the number of $e$-folds, $\phi^{\prime}=d \phi / d N$ etc. The cost of this approach is that we need to solve the differential equation for $H$ alongside hose for the fields. ${ }^{6}$ All model parameters in (55) are functions of $\mu=\mu\left(H, \phi, \phi^{\prime}\right)$, which needs to be computed numerically in each step. This solution of (24) or (42) determines the overall numerical costs.

The first Friedmann equation is used, together with the field equation, in slow-roll approximation to determine the initial conditions self-consistently:

\footnotetext{
${ }^{6}$ Combined with the fact that the field equation is no longer determined by the classical potential that also governs the potential slow-roll parameters, this constitutes the main change that needs to be made to an inflation solver like multimodecode.
} 


$$
\begin{aligned}
0= & 3 H_{0}^{2} \phi_{0}^{\prime} \\
& +\left(m_{\phi, 0}^{2}+\xi_{\phi, 0} 6 H_{0}\left(H_{0}^{\prime}+2 H_{0}\right)\right) \phi_{0}+\frac{1}{6} \lambda_{\phi, 0} \phi_{0}^{3}, \\
3 H_{0}^{2}= & \frac{\frac{1}{2} H_{0}^{2} \phi_{0}^{\prime 2}+\frac{1}{2} m_{\phi, 0}^{2} \phi_{0}^{2}+\frac{1}{24} \lambda_{\phi, 0} \phi_{0}^{4}+6 H_{0}^{2} \phi_{0}^{\prime} \phi_{0} \xi_{\phi, 0}}{1-\phi_{0}^{2} \xi_{\phi, 0}}, \\
H_{0}^{\prime}= & \frac{H_{0}^{-1}}{48\left(\xi_{\phi, 0}\left(6 \xi_{\phi, 0}-1\right) \phi_{0}^{2}+1\right)} \\
& \times\left[1 2 H _ { 0 } ^ { 2 } \left(6 \xi_{\phi, 0}\left(1-8 \xi_{\phi, 0}\right) \phi_{0}^{2}\right.\right. \\
& \left.-4 \xi_{\phi, 0} \phi_{0} \phi_{0}^{\prime}+\left(4 \xi_{\phi, 0}-1\right) \phi_{0}^{\prime 2}-6\right) \\
& \left.+\phi_{0}^{2}\left(\lambda_{\phi, 0}\left(1-8 \xi_{\phi, 0}\right) \phi_{0}^{2}+m_{\phi, 0}^{2}\left(12-48 \xi_{\phi, 0}\right)\right)\right] .
\end{aligned}
$$

Given the initial model parameters and an initial value for the field, $\phi_{0}$, this nonlinear algebraic system determines $H_{0}, \phi_{0}^{\prime}$, and $H_{0}^{\prime}$. It can be solved explicitly, but we solve it numerically for accuracy reasons.

The determination of consistent slow-roll initial conditions, as well as that of the renormalization scale, requires solving nonlinear systems of equations. We have linked the Multimodecode package with Mathematica's WSTP (former MathLink library). This allows us to reach the level of precision required to not pollute Multimodecode's adaptive solving of the system of differential equations that govern inflation and to expose the typically small quantum effects. We can perform these computations at arbitrary precision and need not re-express the lengthy quantum contributions in numerically stable form for the many possible hierarchies of the parameters.

Throughout the paper, we have chosen flat prior distributions for the logarithms of all model parameters at $\mu=\mu_{0}$, each on the intervals quoted in the sections. This is consistent with the approach of [33], but with larger intervals, promoting statistical coverage of a broad range of scales. The choice of logarithmic priors does not necessarily emphasize the quantum effects which are typically bigger for larger couplings. However, it better accounts for the theoretical uncertainty of those parameters in generic inflationary models. Furthermore, even in the case of large couplings, the size of quantum corrections stays typically below the level of a few percent, as we exemplified in Sec. VA. For the field $\phi$, we generate initial values on the interval $[2 ; 30]$ obeying a flat nonlogarithmic distribution. As the pivot scale $k_{*}$, we take the value $k_{*}=0.002$ everywhere. Here, $N_{*}$ varies on the flat prior $[50 ; 60]$ throughout. To determine the end of inflation, we use the condition $\epsilon_{1}=1$. Sampled parameter sets for which not sufficiently many $e$-folds are achieved or for which inflation continues indefinitely are ignored.

Based on the obtained numerical values for the observables $A_{s}, n_{s}$, and $r$, we estimate their multivariate distribution $P\left(A_{s}, n_{s}, r\right)$ and marginalize it with a Gaussian for $\log \left(10^{10} A_{s}\right)$, centered at the Planck experimental value, to obtain $P\left(n_{s}, r\right)$. The confidence regions in Figs. 1, 4, and 10 are given by the contour lines for the values $P_{\alpha}$ $(\alpha=95.45 \%, 68.27 \%)$, for which

$$
\int_{-\infty}^{+\infty} \theta\left(P\left(n_{s}, r\right)-P_{\alpha}\right) P\left(n_{s}, r\right) d n_{s} d r=\alpha .
$$

As explained, the parameters are constrained by the requirement for successful inflation. To perform a Bayesian parameter estimation, a Monte Carlo sampling method would need to be employed, given the high dimensionality of the parameter space. In view of the moderate influence of the quantum contributions in the considered models, and the entailed additional numerical complexities, we have not attempted this in the present work, relying on information from scatter plots. Some of these are displayed above.

\section{CONCLUSION}

We have studied quantum corrections to inflation and their impact in ruling certain models in and out of the Planck-allowed region in $n_{s}-r$ space. Specifically, we considered a single-field model with quadratic and quartic terms, coupled nonminimally to gravity as well as a twofield model in which the second field has a portal coupling with the inflaton field. We simplified the computations by taking the second field to be in its vacuum, mimicking spectator fields coupled to the Standard Model. The quantum corrections enter in the form of the RG running of the model parameters and quantum corrections to the energy momentum tensor that contribute to field and Friedmann equations.

The size of the latter depends on the choice of the renormalization scale. We show that they can be made zero in the single-field and very small in the two-field case, leaving effectively only the RG running to be considered. Although not the truly optimal choice of $\mu$, we argued that the precise value was not crucial. For the single-field model, the smallness of the inflaton self-coupling made the running negligible altogether, whereas in the two-field model the running was still sizeable.

At the classical level, we confirmed that nonminimal couplings to gravity can bring the considered models into agreement with Planck measurements. In order to obtain an unbiased statement about the relevance of the quantum corrections caused by the RG running of the parameters, we sampled the model parameters on broad intervals compatible with existing constraints. We found that the quantum corrections are negligible in the single-field case.

In the second case with two fields, we found that the RG running can influence the trajectories of the field notably if the portal coupling takes large enough values. However, the overall effect on the observables does hardly affect the 
contours of the posterior probability distribution for the observables. It seems that individual trajectories are swapped around inside the contours, without substantially altering them. Interestingly, we found that large values of the portal coupling tend to ruin inflation altogether, putting a limit of $g_{0}<10^{-4}$ up to details of the scale $\mu_{0}\left(\phi_{0}\right)$ where $g_{0}$ is introduced. This bound also restricts the size of the quantum corrections.

In conclusion, a correct and reliable implementation and analysis of quantum corrections to inflation involves considering RG running, allowing for a time- and fielddependent RG scale, including curvature corrections and imposing observational limits on $A_{s}$. We find that $\phi^{2}+\phi^{4}$ inflation coupled to a spectator field is as disfavored at the quantum level as for the classical approximation. We did not do explicit scans for $\xi_{0} \neq 0$ for the two-field model but suspect that a similar conclusion may apply.

The obvious next steps include adapting our procedure to other models of inflation, as well as including IR effects arising from resummations of diagrams (see, for instance, [21]). Of particular interest would be to consider relaxing the semiclassical approach to include both scalar and tensor metric degrees of freedom in our quantum treatment [23]. This poses a number of other challenges to do with the running of gravitational couplings and renormalizability.

\section{ACKNOWLEDGMENTS}

M.H., A.H., and A.T. are supported by the Villum Foundation Grant No. YIP/VKR022599.
[1] E. D. Stewart, Phys. Lett. B 391, 34 (1997).

[2] L. Covi and D. H. Lyth, Phys. Rev. D 59, 063515 (1999).

[3] L. Covi, D. H. Lyth, and L. Roszkowski, Phys. Rev. D 60, 023509 (1999).

[4] T. Inagaki, R. Nakanishi, and S. D. Odintsov, Astrophys. Space Sci. 354, 2108 (2014).

[5] T. Inagaki, R. Nakanishi, and S. D. Odintsov, Phys. Lett. B 745, 105 (2015).

[6] S. D. Odintsov, Phys. Lett. B 306, 233 (1993).

[7] A. Tranberg, J. High Energy Phys. 11 (2008) 037.

[8] J. Serreau, Phys. Rev. Lett. 107, 191103 (2011).

[9] K. Enqvist, R. N. Lerner, and O. Taanila, J. Cosmol. Astropart. Phys. 12 (2011) 016.

[10] D. P. George, S. Mooij, and M. Postma, J. Cosmol. Astropart. Phys. 11 (2012) 043.

[11] B. Garbrecht, F. Gautier, G. Rigopoulos, and Y. Zhu, Phys. Rev. D 91, 063520 (2015).

[12] B. Garbrecht and G. Rigopoulos, Phys. Rev. D 84, 063516 (2011).

[13] D. P. George, S. Mooij, and M. Postma, J. Cosmol. Astropart. Phys. 02 (2014) 024.

[14] F. Gautier and J. Serreau, Phys. Rev. D 92, 105035 (2015).

[15] A. Bilandzic and T. Prokopec, Phys. Rev. D 76, 103507 (2007).

[16] F. Bezrukov, A. Magnin, M. Shaposhnikov, and S. Sibiryakov, J. High Energy Phys. 01 (2011) 016.

[17] T. Markkanen and A. Tranberg, J. Cosmol. Astropart. Phys. 11 (2012) 027.
[18] T. Markkanen and A. Tranberg, J. Cosmol. Astropart. Phys. 08 (2013) 045.

[19] M. Herranen, T. Markkanen, S. Nurmi, and A. Rajantie, Phys. Rev. Lett. 113, 211102 (2014).

[20] K. Enqvist and M. Karciauskas, J. Cosmol. Astropart. Phys. 02 (2014) 034.

[21] M. Herranen, T. Markkanen, and A. Tranberg, J. High Energy Phys. 05 (2014) 026.

[22] T. Markkanen, Phys. Rev. D 91, 124011 (2015).

[23] M. Herranen, A. Osland, and A. Tranberg, Phys. Rev. D 92, 083530 (2015).

[24] R. Allahverdi, K. Enqvist, J. Garcia-Bellido, A. Jokinen, and A. Mazumdar, J. Cosmol. Astropart. Phys. 06 (2007) 019.

[25] K. Enqvist and A. Mazumdar, Phys. Rep. 380, 99 (2003).

[26] L. C. Price, J. Frazer, J. Xu, H. V. Peiris, and R. Easther, J. Cosmol. Astropart. Phys. 03 (2015) 005.

[27] A. R. Liddle, P. Parsons, and J. D. Barrow, Phys. Rev. D 50, 7222 (1994).

[28] T. Chiba and M. Yamaguchi, J. Cosmol. Astropart. Phys. 01 (2009) 019.

[29] T. Chiba and M. Yamaguchi, J. Cosmol. Astropart. Phys. 10 (2008) 021.

[30] J. R. Morris, Classical Quantum Gravity 18, 2977 (2001).

[31] D. F. Torres, Phys. Lett. A 225, 13 (1997).

[32] H. Noh and J. c. Hwang, Phys. Lett. B 515, 231 (2001).

[33] P. A. R. Ade et al. (Planck Collaboration), Astron. Astrophys. 594, A20 (2016). 